\title{
Preferenda thermiques des champignons associés à Ips sexdentatus Boern. et Tomicus piniperda L. (Coleoptera : Scolytidae)
}

\author{
F. Lieutier et A. Yart
}

INRA, station de zoologie forestière Ardon, 45160 Olivet, France

(reçu le 3 avril 1989; accepté le 5 juin 1989)

Résumé - Trois espèces de champignons associées à $T$. piniperda et deux espèces associées à I. sexdentatus sont cultivées au laboratoire sur malt-agar, à différentes températures. Leurs vitesses de croissance et leurs preferenda thermiques sont comparés, puis discutés en liaison avec les réactions de défense de l'arbre hôte et les époques de vol et d'attaque des deux Scolytides-hôtes.

Leptographium wingfieldii - Ophiostoma minus - Hormonema dematioides - Ophiostoma ips - Ophiostoma brunneo-ciliatum - température - agressivité

Summary - Temperature preferenda of the fungi associated with Ips sexdentatus Boern. and Tomicus piniperda L. (Coleoptera : Scolytidae). Five species of fungi, Leptographium wingfieldii, Ophiostoma minus and Hormonema dematioides associated with T. piniperda, Ophiostoma ips and Ophiostoma brunneo-ciliatum associated with I. sexdentatus were grown on a malt-agar medium in the laboratory, under several different temperatures. Their speeds of growth and their temperature preferenda were compared and discussed in relation to the defense reactions of the host tree and to the dates of flight and tree attack of the beetles. According to the present findings, $\mathrm{L}$. wingfieldii, $\mathrm{O}$. ips and $\mathrm{O}$. brunneo-ciliatum seem better adaptered to their host than $\mathrm{O}$. minus and H. dematioides.

Leptographium wingfieldii - Ophiostoma minus - Hormonema dematioides - Ophiostoma ips - Ophiostoma brunneo-ciliatum - temperature - aggressiveness

\section{INTRODUCTION}

On sait depuis longtemps que la plupart des Scolytides transportent les spores de divers champignons phytopathogènes
(Graham, 1967; Francke-Grossman, 1967; Whitney, 1982). Ce n'est toutefois que récemment que de nombreux entomologistes forestiers s'accordent à attacher à ces microorganismes un rôle prépondé- 
rant dans les interrelations arbres-insectes et en particulier dans les mécanismes d'installation des Scolytides sur les conifères. On leur attribue ainsi la responsabilité de l'induction des réactions de défense dont dépendrait le succès ou l'échec des attaques, et même souvent la responsabilité de la mort des arbres attaqués (Berryman, 1972; Safranyik et al., 1975; Christiansen \& Horntvedt, 1983; Horntvedt et al., 1983; Raffa \& Berryman, 1983; Christiansen et al., 1987).

Dans le cadre d'un programme de travail sur les Scolytides ravageurs du pin sylvestre, nous avons entrepris d'examiner le fonctionnement des relations entre cet arbre et deux Scolytides de grand intérêt économique en Europe, Ips sexdentatus Boern. et Tomicus piniperda L. Notre but est, dans un premier temps, de définir la place et le rôle des différents protagonistes. Nous avons déjà pu dresser un inventaire des champignons associés aux deux Scolytides, établir leur fréquence d'association avec ces insectes et comparer leur agressivité pour le pin sylvestre (Lieutier et al., 1989). Leur mode de transport sur l'insecte hôte a également été examiné (Lévieux et al., 1989).

Afin de mieux définir les liens qui unissent ces champignons avec les Scolytides qui les transportent, nous nous proposons ici, grâce à des tests en laboratoire, de comparer leurs cinétiques de croissance et de déterminer leurs exigences thermiques. Dans le domaine des relations Conifères-Scolytides, ces aspects n'ont encore jamais été abordés en liaison avec l'agressivité des champignons pour l'arbre, les capacités de défense de celuici ou les exigences thermiques des insectes vecteurs. Cinq espèces ont été retenues d'après leur fréquence d'association avec les insectes et leur agressivité pour le pin sylvestre : Leptographium wingfieldii Morelet, Ophiostoma minus Hedgc., Hormonema dematioides Lagerb et Melin associés à T. piniperda et Ophiostoma ips (Rumb.) Nannf. et Ophiostoma brunneo-ciliatum Math.-K associés à $I$. sexdentatus.

\section{MATÉRIELS ET MÉTHODES}

Les champignons récoltés en forêt d'Orléans proviennent à l'origine d'isolements réalisés à partir d'insectes et de galeries de forage (Lieutier et al., 1989). Ils ont été ensuite purifiés par culture monospores et entretenus au laboratoire sur milieu gélosé à $3 \%$ de malt. Les tests ont eu lieu sur le même milieu. Une pastille calibrée de $5 \mathrm{~mm}$ de diamètre, découpée à l'emporte-pièce dans une culture sporulée âgée de 2 semaines, a été déposée mycélium contre gélose, au centre de chaque boite de Petri, placée ensuite à température constante à l'obscurité. Pour chaque champignon, 8 températures ont été testées, de 5 à $40^{\circ} \mathrm{C}$ tous les $5^{\circ} \mathrm{C}$, à raison de 2 à 4 répétitions par champignon et par température. Tous les jours, l'extension de chaque colonie a été appréciée par la mesure de deux diamètres orthogonaux entre eux, dont la moyenne a ensuite été calculée après qu'a été soustrait de chacun d'eux le diamètre de l'implant. La date de début de fructification (anamorphes) a aussi été notée.

\section{RÉSULTATS (Fig. 1)}

Pour 0 . ips, 0 . brunneo-ciliatum et 0 . minus, la rapidité maximale de croissance est obtenue à $30^{\circ} \mathrm{C}$. $O$. minus est le plus rapide, et sa croissance est déjà très importante à $5^{\circ} \mathrm{C}$. $O$. ips et $O$. brunneociliatum ont des vitesses de croissance voisines, mais le premier paraît légèrement plus rapide et supporte mieux les hautes températures. Ainsi, alors que $O$. brunneo-ciliatum devient blanchâtre après quelques jours à $35^{\circ} \mathrm{C}$, dégénère complètement et ne parvient pas à coloniser la totalité de la boîte, $O$. ips ne subit à cette température qu'un ralentissement de 


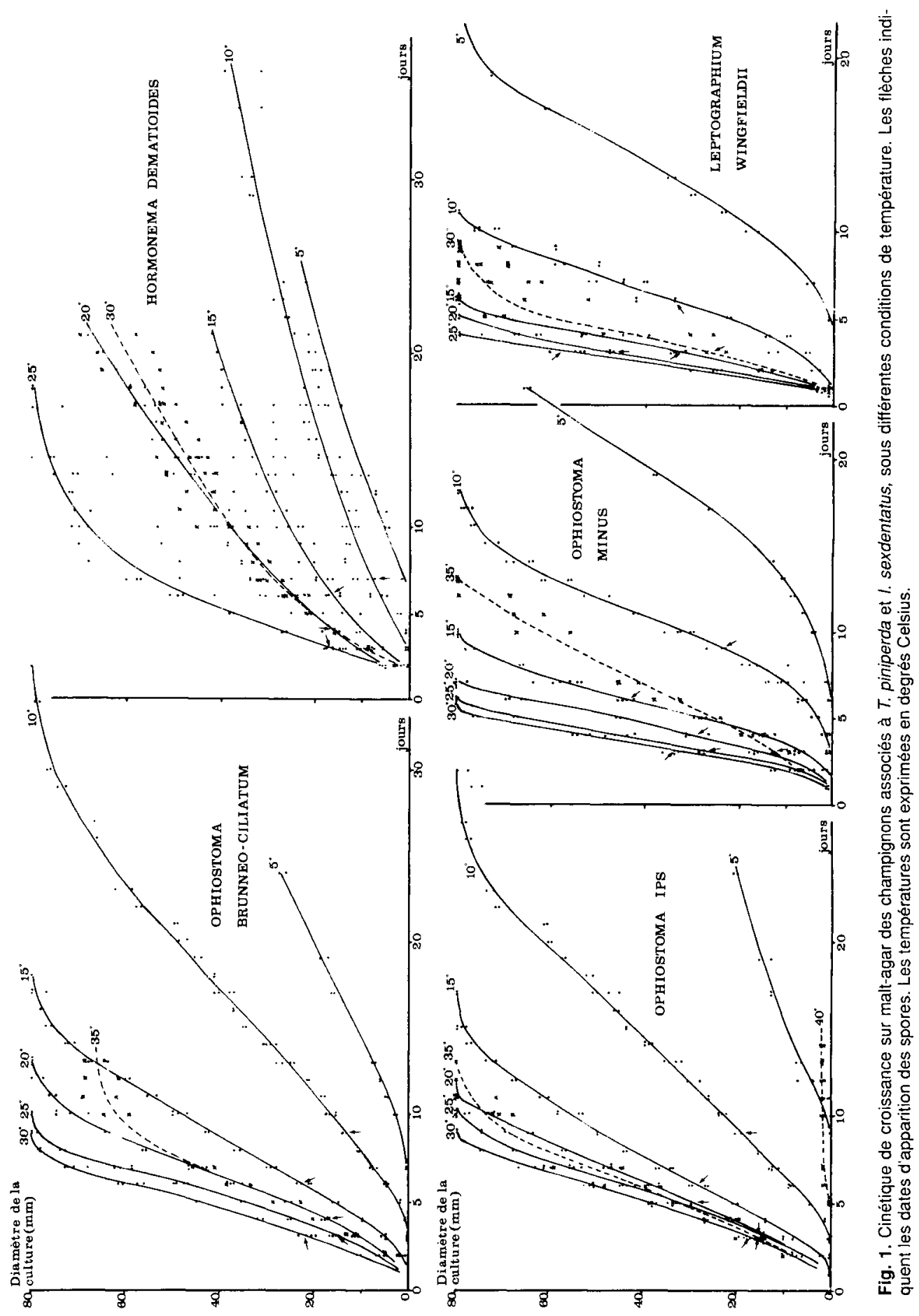


croissance. En outre, $O$. brunneo-ciliatum ne pousse pas du tout à $40^{\circ} \mathrm{C}$, alors que $O$. ips survit.

$L$. wingfieldii et $H$. dematioides ont une rapidité maximale de croissance à $25^{\circ} \mathrm{C}$; ils ne poussent pas du tout au-delà de $30^{\circ} \mathrm{C}$. Entre $5^{\circ} \mathrm{C}$ et $25^{\circ} \mathrm{C}, \mathrm{L}$. wingfieldii est le plus rapide de tous les champignons testés; sa vitesse de croissance à $5^{\circ} \mathrm{C}$ et $10^{\circ} \mathrm{C}$ est même remarquable. $H$. dematioides croît en revanche très lentement à toutes les températures.

Les dates de début de fructification concordent avec les observations précédentes, mais aucun champignon ne fructifie avant 3 jours, quelles que soient sa vitesse de croissance et la température. Aucun non plus ne fructifie à $5^{\circ} \mathrm{C}$.

\section{INTERPRÉTATION ET DISCUSSION}

L'agressivité des champignons avait précédemment été jugée élevée quand ceux$c i$ induisaient une forte réaction de défense de la part de l'arbre et se montraient capables d'une croissance importante à l'intérieur de cette réaction (Lieutier et al., 1989). Or, le classement des champignons en fonction de leurs vitesses de croissance sur malt-agar, donc en l'absence de réactions, se révèle être le même que pour leur agressivité. Les différences de croissance observées après inoculation sur arbre ne peuvent donc être attribuées aux réactions de défense du végétal, ou à une éventuelle tolérance plus ou moins marquée à la résine. Elles correspondent simplement à des caractéristiques intrinsèques des champignons. On peut se demander alors si l'importance de la réaction de défense de l'arbre inoculé ne dépend pas uniquement de la vitesse de croissance du champignon.
Les vitesses de croissance obtenues à $20^{\circ} \mathrm{C}$ pour les 3 Ophiostoma sont nettement supérieures à celles indiquées à $22{ }^{\circ} \mathrm{C}$ par Upadhyay (1981), ce qui peut s'expliquer en particulier par la nature différente des milieux de culture. La comparaison des courbes de croissance aux différentes temperratures montre en outre que $L$. wingfieldii supporte mal les hautes températures, son optimum thermique étant de $25^{\circ} \mathrm{C}$, mais qu'il supporte en revanche très bien les basses températures. Or, ce champignon provient de $T$. piniperda, dont la période d'envol et d'attaque, donc d'introduction dans l'arbre des champignons qu'il transporte, a toujours lieu précocément dans l'année, fin février-début mars, à une époque où les températures sont peu élevées, le seuil thermique d'envol de cet insecte étant de $12{ }^{\circ} \mathrm{C}$ (Chararas, 1962; Vallet, 1982). En revanche $O$. ips et $O$. brunneo-ciliatum, isolés d'l. sexdentatus, poussent et fructifient mal à basse température, ce qui est conforme à la localisation en début mai et fin juillet des deux périodes d'attaque de ce scolyte, dont le seuil thermique d'envol est de $18{ }^{\circ} \mathrm{C}$ (Chararas, 1962; Vallet, 1982). Ces résultats vont dans le sens d'une bonne adaptation de ces 3 champignons aux scolytes qui les transportent.

O. minus, cornme L. wingfieldii, provient de $T$. piniperda mais, bien que supportant les basses températures presque aussi bien que $L$. wingfieldii, il possède un optimum thermique de croissance voisin de $30^{\circ} \mathrm{C}$, comme les 2 Ophiostoma associés à $l$. sexdentatus. L'association entre $O$. minus et son insecte hôte paraît ainsi moins étroite que dans les trois cas précédents. Ces observations sont à rapprocher de l'irrégularité, à la fois dans le temps et dans l'espace, de la fréquence d'association de $O$. minus avec $T$. piniperda, qui nous avait conduit à mettre en doute la fonctionnalité de cette association dans les mécanismes d'installation de ce scoly- 
te sur pin sylvestre (Lieutier et al., 1989; Piou et Lieutier, 1989). Quant à $H$. dematioides, bien que possédant un optimum de croissance à $25^{\circ} \mathrm{C}$, il pousse très mal à basse température, ce qui rend peu probable son association réelle avec l'un ou l'autre des deux Scolytides considérés.

Les tests rapportés ici nous ont donc fourni des informations utiles sur les exigences thermiques des champignons associés aux deux Scolytides que nous étudions. Des expérimentations complémentaires sur arbre vivant sont nécessaires avant de conclure à la réalité des associations évoquées.

\section{RÉFÉRENCES}

Berryman A. (1972) Resistance of conifers to invasion by bark Beetle-fungus associations. Bio Science, 22, 599-601

Chararas C. (1962) Etude biologique des Scolytides des Coniferes. Le Chevalier Ed., Paris, $556 \mathrm{pp}$.

Christiansen E. \& Horntvedt R. (1983) Combined Ips/Ceratocystis attack on Norway spruce, and defensive mechanisms of the trees. $Z$. Angew. Entomol., 96, 110-118

Christiansen E., Waring R.H. \& Berryman A.A. (1987) Resistance of conifers to bark beetle attack : searching for general relationship. For. Ecol. Manag., 22, 89-106

Francke-Grossman H. (1967) Ectosymbiosis in wood-inhabiting insects. Pages 141-205 in : Symbiosis, S.M. Henry edit., vol. 2, 443 pp., Acad. Press, N.Y.

Graham K. (1967) Fungal-insect mutualism in trees and timber. Ann. Rev. Entomol. 12, 105126

Horntvedt R., Christiansen E., Solheim H. \& Wang S.G. (1983) Artificial inoculation with lps typographus associated blue-stain fungi can kill healthy Norway spruce trees. Medd. fra Norsk Inst. For. skogf., 38, 1-20

Lévieux J., Lieutier F., Moser J.C. \& Perry T. (1989) Transportation of phytopathogenic fungi by the bark beetle lps sexdentatus boern. and associated mites. J. Appl. Entomol. 108, 1-11

Lieutier F., Yart A., Garcia J., Ham M.C., Morelet M. \& Lévieux J. (1989) Champignons phytopathogènes associés à deux coléoptères Scolytidae du pin sylvestre (Pinus sylvestris L.) et étude préliminaire de leur agressivité envers lhôte. Ann. Sci. For. 46, 201-216

Piou D. \& Lieutier F. (1989) Observations symptomatologiques et rôles possibles d'Ophiostoma minus [Hedgc.] (ascomytes : Ophiostomatales) et de Tomicus piniperda L. (Coleoptera : Scolytidae) dans le dépérissement du pin sylvestre en forêt d'Orléans. Ann. Sci. For., 46, 3953

Raffa K.F. \& Berryman A.A. (1983) The role of host resistance in the colonization behavior and ecology of bark beetles. (Coleoptera : Scolytidae). Ecol. Monog., 53, 27-49

Safranyik L., Shrimpton D.M. \& Whitney H.S. (1975) An interpretation of the interaction between lodgepole pine, the mountain pine beetle, and its associated blue stain fungi in western Canada. In : Management of Lodgepole Pine Ecosystems. D.M. Baumgartner Ed., Pullman; Wash. State Univ. Coop. Ext. serv., 406-428

Upadhyay H.P. (1981) A monograph of Ceratocystis and Ceratocystiopsis. The Univ. of Georgia Press. Athens. $176 \mathrm{pp}$.

Vallet E. (1982) Données bioécologiques récentes sur trois insectes ravageurs responsables du dépérissement du pin sylvestre en région Centre : Tomicus piniperda, Ips acuminatus et lps sexdentatus (Col., Scolytidae). Bull. Institut Ecol. Appl., Orléans, 1-2, 3-41

Whitney H.S. (1982) Relationships between bark beetles and symbiotic organisms. In : Bark beetles in North american Conifers. (Mitton J.B., Sturgeon K.B. Eds.) University of Texas Press, Austin, 527 pp, 183-211

Wright E. (1933) A cork-borer method for inoculating trees. Phytopathol., 23, 487-488 